\section{AB0867 PEGLOTICASE RE-TREATMENT AFTER A GAP IN THERAPY: DATA FROM TWO PHASE III TRIALS AND AN OPEN-LABEL EXTENSION STUDY}

H.S. Baraf ${ }^{1}$, A. Morton ${ }^{2}$, B. LaMoreaux ${ }^{3}$, J. Kent ${ }^{3} .{ }^{1}$ Medicine, George Washington University, Washington DC; ${ }^{2}$ Internal Medicine, Michigan State University School of Osteopathic Medicine, East Lansing; ${ }^{3}$ Medical Affairs, Horizon Pharma, Lake Forest, United States

Background: Pegloticase is a PEGylated recombinant uricase approved in the US for treating adult patients with chronic refractory gout. As a biologic medication, pegloticase is administered intravenously every 2 weeks. It is currently not known whether a gap in the standard biweekly regimen can be tolerated or would be associated with a loss of efficacy. During the pivotal trial testing of pegloticase, some patients experienced a delay between participation in the randomized controlled trial (RCT) and the open-label extension (OLE) that followed. Analysis of the clinical impact of this gap was carried out to understand whether therapeutic benefit would be affected.

Objectives: The objective of this analysis was to determine, among pegloticase responders, the effect of a 28 or more day gap between doses of pegloticase on the subsequent urate lowering response and frequency of infusion reactions.

Methods: These analyses utilized results from two RCTs of pegloticase and a 2-year OLE. In the RCTs, 36 of 85 patients, who were dosed with pegloticase every two weeks, were classified as responders (persistent urate lowering during intensive monitoring at 3 and 6 months of the RCT), and went on to enroll in the OLE and receive additional doses of pegloticase. Of these 36 patients, 14 had a gap between pegloticase doses of more than 28 days.

Results: Among the 14 patients with a gap of more than 28 days between doses of pegloticase therapy the length of the gap ranged from 34 to 167 days (mean $=72.5$ days, median $=59.5$ days). Of these 14 patients, 8 received pegloticase on an every-4-week dosing schedule in the OLE and 6 remained on every-2-week dosing. Ten of the 14 patients maintained their serum urate level $<6 \mathrm{mg} / \mathrm{dL}$ in the OLE. Five of 6 that remained on every 2 week dosing and 5 of 8 that went to every 4 week dosing continued to have serum urate $<6 \mathrm{mg} / \mathrm{dL}$. (see table 1). By logistic regression analysis, the length of the gap had no significant effect on the subsequent urate lowering effect of pegloticase. Of the fourteen patients with a gap in pegloticase therapy, $2(14 \%)$ had infusion reactions during a total of 632 infusions in the OLE yielding a re-treatment IR rate of $0.32 \%$.

Table 1. Continued responder proportions and infusion reaction rates by OLE dosing group

\begin{tabular}{lcccc}
\hline Dosing Group & Total & Continued Responders & Percentage & Infusion Reaction Rate \\
\hline Combined & 14 & 10 & $71 \%$ & 0.32 \\
Every 2 weeks & 6 & 5 & $83 \%$ & 0.30 \\
Every 4 weeks & 8 & 5 & $62.5 \%$ & 0.34 \\
\hline
\end{tabular}

Conclusions: The majority of patients in this limited dataset who were previously responders to pegloticase dosed every 2 weeks continued to maintain a serum urate lowering response to pegloticase after a gap in therapy. Infusion reactions during re-treatment occurred in 2 patients with a re-treatment infusion reaction rate of $0.32 \%$.

References:

[1] Sundy, J.S., Baraf, H.S.B., Yood, R.A., et al. Efficacy and tolerability of pegloticase for the treatment of chronic gout in patients refractory to conventional treatment, JAMA, 2011;306(7):711-720.

[2] Becker, M.A., Baraf, H.S.B., Yood, R.A., et al. Long-term safety of pegloticase in chronic gout refractory to conventional treatment, Ann Rheum Dis, 2013;72:1469-1474.

[3] Lipsky, P.E., Calabrese, L.H., Kavanaugh, A., et al. Pegloticase immunogenicity: the relationship between efficacy and antibody development in patients treated for refractory chronic gout, Arthritis Research \& Therapy, 2014;16:R60. Disclosure of Interest: H. Baraf Consultant for: Horizon Pharma, Takeda, Ironwood, A. Morton Consultant for: Pfizer, Sanofi Regeneron, Ironwood, Horizon, Speakers bureau: Prizer, Amgen, Abbvie, Janssen, Celgene, Horizon, Novartis, Ironwood, B. LaMoreaux Employee of: Horizon Pharma, J. Kent Employee of: Horizon Pharma

DOI: 10.1136/annrheumdis-2017-eular.5365

\section{AB0868 IS IT APPROPRIATE TO DISCONTINUE COLCHICINE THERAPY IN GOUT PATIENTS IN REMISSION?}

Y. Özdem İnan ${ }^{1}$, N. Alpay Kanıtez ${ }^{2}$, S. Çelik ${ }^{2}$, S. Yılmaz Öner ${ }^{2}$, C. Bes ${ }^{2}$ ${ }^{1}$ Bakırköy Dr Sadi Konuk Training and Research Hospital, Istanbul, Turkey; ${ }^{2}$ Rheumatology, Bakırköy Dr Sadi Konuk Training and Research Hospital, Istanbul, Turkey

Background: Colchicine can reduce the risk of recurrent attacks and it is an appropriate first-line gout attack prophylaxis therapy. There is limited data in the literature about duration of colchicine treatment in patients with gout disease.

Objectives: In this study, we aimed to investigate the frequency of gout attacks between patients with colchicine treatment and patients who are colchicine terminated.

Methods: 54 gout patients under colchicine treatment were enrolled to the study. The subjects were divided into 2 groups. Group $1(n=19)$ was consisted of patients whom colchicine therapy was terminated because of remission for three months; group $2(n=35)$ was consisted of patients on colchicine treatment. Groups were compared according to the existing of newly gout attacks after the 3rd month Baseline and the third months of the uric acid levels were also analyzed in each group.

Results: The body mass indexes, the disease intervals, the number of the attacks, and the duration of the attacks were not significantly different ( $p>0.05$ for all) (Table 1). Compared to the group 2, the newly gout attacks were not found to be different in group 1 for 3 months ( $p>0.05$ ) (Table 1). The uric acid levels were significantly decreased both in two groups (both $p<0.05$ ) (Table 2).

Table 1. Demographical and clinical characteristics of patients

\begin{tabular}{lccc}
\hline Patient variables & Group 1 $(\mathrm{n}=19)$ & Group 2 $(\mathrm{n}=35)$ & $\mathrm{p}$ \\
\hline Gender, F/M & $0 / 19$ & $12 / 23$ & 0.040 \\
Age, years & $53.36 \pm 14.06$ & $62.11 \pm 12.72$ & 0.024 \\
BMl & $28.22 \pm 3.57$ & $30.29 \pm 5.63$ & 0.153 \\
Uric acid, mg/dL & $7.40 \pm 1.30$ & $8.21 \pm 1.92$ & 0.108 \\
Disease age, months & $111.63 \pm 172.70$ & $53.48 \pm 59.75$ & 0.170 \\
Number of the attacks, one year & $2.68 \pm 2.35$ & $3.37 \pm 3.75$ & 0.413 \\
Duration of the attacks, days & $16.73 \pm 16.32$ & $10.91 \pm 14.50$ & 0.202 \\
Newly attacks in 3 months & $5 / 14$ & $7 / 28$ & 0.734 \\
\hline
\end{tabular}

attacks in 3 months

Table 2. Before and after treatment comparisons of the uric acid levels

\begin{tabular}{lccc}
\hline Variables & Baseline & 3rd month & $\mathrm{p}$ \\
\hline Group1 & $7.40 \pm 1.30$ & $6.47 \pm 1.19$ & 0.008 \\
Group 2 & $8.21 \pm 1.92$ & $6.74 \pm 1.70$ & $<0.001$ \\
\hline
\end{tabular}

Conclusions: The colchicine is the mainstay treatment for gout disease. It not only prevents the attacks of the gouts also prevents the development of uric acid nephropathy that exaggerates the kidney disease in gout patients. This study showed that, in gout patients in remission, termination of the colchicine treatment does not affect the development of the new attacks as shown similar attacks were also observed in non-terminated patients.

Disclosure of Interest: None declared

DOI: 10.1136/annrheumdis-2017-eular.1423

\section{AB0869 STRESS FRACTURES, A RETROSPECTIVE STUDY OF 325 FRACTURES IN 227 PATIENTS IN RHEUMATOLOGY PRACTICE BETWEEN 2000 AND 2015}

C. Nourisson, J.-J. Dubost, B. Pereira, A. Tournadre, S. Malochet-Guinamand, M. Soubrier. CHU Clermont Ferrand, Clermont-Ferrand, France

Background: Stress fractures are common in both young and active patients (fatigue fracture) as well as in elderly osteoporotic patients (insufficiency fractures). Objectives: The aim of this study was to describe characteristics of stress fractures treated in rheumatology at the Clermont-Ferrand University Hospital, to compare them according to their anatomic location (pelvis, lower limb or feet/ankle) and their diaphyseal or metaphyseal/epiphyseal location.

Results: Between 01/01/2000 and 12/31/2015, 325 fractures were identified in 227 patients divided in 176 women (including 116 postmenopausal women) and 51 men. Population averaged age was 65.2 years [15-99 years]. 142 (43.7\%) fractures occurred at pelvis, $93(28.6 \%)$ at lower limbs and $87(26.8 \%)$ at feet and ankles. The fracture was spontaneous in $63.6 \%$ of cases. History of pain was recorded in $92.3 \%$ with more frequently $(90.3 \%)$ mechanical pain. Diagnostic delay was on average 70 days.

In 51 patients $(22.5 \%)$ an orthopedic factor could have promoted stress fracture. 54 patients $(23.8 \%)$ had a history of chronic inflammatory rheumatism, $28(12.3 \%)$ of cancer, and $20(8.8 \%)$ had chronic renal failure.

Among the possible iatrogenic factors, 51 patients $(23.4 \%)$ received oral corticosteroid therapy, $20(9.2 \%)$ methotrexate, 59 (27\%) proton pump inhibitor and $17(7.8 \%)$ serotonin reuptake inhibitor. 49 patients $(22.5 \%)$ already had vitamin-calcium supplementation, 37 (17\%) received biphosphonate therapy. $56.6 \%$ were osteoporotic and $29.5 \%$ were osteopenic.

First-line medical imaging was in $87 \%$ conventional radiography, positive in $37 \%$. 106 patients $(46.7 \%)$ performed bone scan with a sensibility of $99 \%$. MRI sensibility $(n=65,28.7 \%)$ was $88 \%$ and CT-scan sensibility $(n=47,20.7 \%) 65.2 \%$. Compared to other sites, pelvic fractures were more frequent $(p<0.001)$ and occurred more frequently in postmenopausal women $(p=0.03)$. These patients were older $(p<0.001)$ and had more fracture history $(p=0.006)$. Hip bone mineral density $(p=0.03$ ) was lower, and osteoporosis prevalence higher $(p=0.004)$.

Pelvic fractures occurred more frequently after fall $(p<0.001)$, lower limb fractures spontaneously $(p=0.03)$ and those of the feet-ankles group after an unusual activity $(p<0.001)$. Patients from metaphyseal-epiphyseal fracture group were older $(p=0.05)$ than those from the diaphyseal fractures group and delay in completing complementary examinations was longer $(p=0.04)$.

Conclusions: In conclusion, pelvic stress fractures are more frequent in a rheumatology department and suggest insufficiency fracture. Stress fracture should be more often suspected when mechanical pain of the pelvis and lower limbs occurs, even spontaneously. Regardless of the site, an assessment of fracture risk factors and bone mineral density evaluation seems necessary.

Disclosure of Interest: None declared

DOI: 10.1136/annrheumdis-2017-eular.6741 